Please do not remove this page

RMIT

UNIVERSITY

\title{
Comment on "Relative variance of the mean squared pressure in multimode media: Rehabilitating former approaches" [J. Acoust. Soc. Am. 136, 2621-2629 (2014)]
}

Davy, John; Weaver, Richard

https://researchrepository.rmit.edu.au/esploro/outputs/9921861773301341/filesAndLinks?institution=61RMIT_INST\&index=null

Davy, J., \& Weaver, R. (2015). Comment on "Relative variance of the mean squared pressure in multimode media: Rehabilitating former approaches" [J. Acoust. Soc. Am. 136, 2621-2629 (2014)]. Journal of the Acoustical Society of America, 137(3), 1598-1601. https://doi.org/10.1121/1.4913276

Document Version: Accepted Manuscript

Published Version: https://doi.org/10.1121/1.4913276

Repository homepage: https://researchrepository.rmit.edu.au

(C) 2015 Acoustical Society of America

Downloaded On 2023/04/26 18:38:40 +1000 
Thank you for downloading this document from the RMIT Research Repository.

The RMIT Research Repository is an open access database showcasing the research outputs of RMIT University researchers.

RMIT Research Repository:http://researchbank.rmit.edu.au/

\section{Citation:}

Davy, J and Weaver, R 2015, 'Comment on "Relative variance of the mean squared pressure in multimode media: Rehabilitating former approaches" [J. Acoust. Soc. Am. 136, 2621-2629 (2014)]', Journal of the Acoustical Society of America, vol. 137, no. 3, pp. 1598-1601.

See this record in the RMIT Research Repository at:

https://researchbank.rmit.edu.au/view/rmit:30162

Version: Accepted Manuscript

Copyright Statement: (C) 2015 Acoustical Society of America

Link to Published Version:

http://dx.doi.org/10.1121/1.4913276 
Comment on "Relative variance of the mean squared pressure in multimode media: rehabilitating former approaches" [J. Acoust. Soc. Am. 136, 2621-2629 (2014)]

\author{
John L. Davy ${ }^{\text {ab }}$
}

School of Applied Sciences, RMIT University, GPO Box 2476V Melbourne, Victoria 3001, Australia

\begin{abstract}
Richard L. Weaver
Department of Physics and Institute for Condensed Matter Theory, University of Illinois at Urbana-Champaign, 1110 West Green Street, Urbana, Illinois 61801, USA
\end{abstract}

Running title: The variance of the transmission function

\footnotetext{
${ }^{a}$ Electronic mail: Author to whom correspondence should be addressed. john.davy@rmit.edu.au

${ }^{\mathrm{b}}$ Also employed at CSIRO Materials Science and Engineering, PO Box 56 Highett, Victoria 3190, Australia.
} 
Models for the statistics of responses in finite reverberant structures, and in particular for the variance of the mean square pressure in reverberation rooms, have been studied for decades. It is therefore surprising that a recent JASA communication has claimed that the literature has gotten the simplest of such calculations very wrong. Monsef et al. ["Relative variance of the mean squared pressure in multimode media: rehabilitating former approaches" J. Acoust. Soc. Am. 136, 2621-2629 (2014)] have derived a modal-based expression for the relative variance that differs significantly from expressions that have been accepted since 1969. This comment points out that the Monsef formula is clearly incorrect, and then for the interested reader, points out the subtle place where they made their mistake.

PACS numbers: 43.55.Cs, 43.55.Br, 43.55.Nd, 43.50.Cb 


\section{INTRODUCTION}

The theory for power variances in reverberant structures and rooms has attracted attention for many decades now. It is clear that at large modal overlap $M=2 \pi n \gamma$, where $n$ is the modal density (modes per unit angular frequency $\omega$ ) and $\gamma$ is the dissipation rate (units of nepers per time) of acoustic amplitude, the underlying modal character ought not be discernible and (timedomain) responses ought be indistinguishable from real Gaussian random processes under an exponential decay envelope. Thus at large overlap the relative variance is unity, i.e. the expected fluctuations in the measured power are comparable to the power itself. These are called Ericson fluctuations in nuclear physics (Legrand et al., 1995).

At more modest overlap the variance is expected to be much larger, and the search for an accurate theory has attracted considerable attention. A variance larger than unity at small $M$ is easily understood if one recalls that, at small $M$, the resonances are relatively distinct. In this case room response varies strongly as the frequency of excitation coincides, or not, with an eigenfrequency of the room. Early theory (Lyon, 1969) based on an assumption of uncorrelated eigenfrequencies suggested the relative variance should be $1+K^{2} / M$ for all $M$, with $K$ related to the statistics of the mode shapes, equal to $\left\langle u^{4}\right\rangle \mid\left\langle u^{2}\right\rangle^{2}$, the ratio of the mean fourth power of a mode amplitude to the square of the mean square.

Davy (1981; 1986; 1987), and Lyon (1969), showed that this formula is modified if the eigenfrequencies are correlated, predicting at large $M, 1+\left(K^{2}-3 / 2\right) / M$ for what Lyon called nearest-neighbor (NN) eigenfrequency repulsion. Weaver (1989a) pointed out that that those NN eigenfrequency correlations lack the long-range rigidity predicted by random matrix theory (Brody et al., 1981). He then showed that the full eigenfrequency correlations derived from the Gaussian Orthogonal Ensemble (GOE) of Random Matrix Theory (RMT) modifies the formula 
further. Like others Weaver obtained $1+K^{2} / M$ at small $M$ (regardless of correlations). But at large $M$ he found that long range spectral rigidity leads to the expression $1+\left(K^{2}-3\right) / M$.

These theoretical predictions are further modified if the modal decay rates $\gamma$ are taken to vary randomly between the modes ("decay curvature") (Burkhardt and Weaver, 1996; Lobkis et al., 2000).

The value of $K$ has also received attention. Conventional RMT predicts that the mode shapes are real Gaussian random processes. To the extent that actual modes are complex, $K$ ought to be less than three. Langley and Brown (2004a; 2004b) and Langley and Cotoni (2005) have argued that $K$ ought be less than three based on realizing that the modes of finite sized orthogonal random matrices will not be Gaussian. Fits of measured variances to theory have suggested values of $K$ between 2.5 and 4 (Langley and Brown, 2004a; b; Langley and Cotoni, 2005; Jacobsen, 2012).

These formulas are readily generalized for the variances and covariances of measurements of power averaged over multiple sources and/or receivers at different working frequencies (Davy, 1981; 1986; 1987).

A distinct theory that does not consider modes but proceeds directly from the random orthogonal dissipative Hamiltonian and includes the effects of decay curvature and complex modes has been suggested also, but it is difficult to calculate with (Rozhkov et al., 2004).

Differences over realistic choices for $K$, the importance of decay curvature, the range of level rigidity and focus on covariances and/or multiple sources and receivers notwithstanding, all theorists seem to be in agreement that the relative variance for power transmission between distant points ought be exactly (for the special and simple case of no eigenfrequency correlations and no decay curvature and real modes and a single source and receiver) $1+K^{2} / M$. It is therefore 
surprising that in a recent communication, Monsef et al. (2014) have suggested that the correct expression for these conditions is $1+\left(K^{2}-2\right) / M$. Either Monsef et al. (2014) have discerned an error that has escaped the attention of others for fifty years, or they have made a mistake. The mystery is compounded further as they, realizing their unusual claim requires corroboration, show numerical Monte Carlo calculations that appear to be in agreement with their formula.

This comment proceeds by first demonstrating that their expression violates a required mathematical inequality and therefore cannot be correct. It then identifies where in their analysis they made their error. It then speculates as to what aspects of their Monte Carlo calculation led to its agreement with the erroneous theory.

\section{RELATIVE VARIANCE OF A MODAL RESPONSE SUM}

Monsef et al.'s analytic derivation starts as do others with an expression equivalent to

$$
p(\mathbf{x}, \omega)=\sum_{m} \frac{u_{m}(\mathbf{x}) u_{m}(\mathbf{s})}{\omega^{2}-\omega_{m}^{2}-2 i \omega \gamma_{m}}
$$

This describes the complex steady state pressure at a position $\mathbf{x}$ due to a harmonic point source at position $\mathbf{s}$. The $u$ are the normal modes (assumed real in the current application). The $\omega_{m}$ are the natural frequencies and $\gamma_{m}$ are the modes' absorption rates. We seek the statistics, in particular the mean square of $|p|$ and the mean fourth power of $|p|$. To estimate these one must make assumptions about the statistics of the $u$ and of the $\omega_{m}$.

The mode shapes $u_{n}$ and $u_{m}$ are traditionally assumed uncorrelated for $n \neq m$, and the positions $\mathbf{x}$ and $\mathbf{s}$ are assumed such that $u_{m}(\mathbf{x})$ is uncorrelated with $u_{m}(\mathbf{s})$. It is convenient to take the $u_{n}$ to be centered real Gaussian random numbers. A key quantity, it transpires, is the ratio of

the mean fourth power of $u$ to the square of the mean square: $K=\left\langle u^{4}\right\rangle \mid\left\langle u^{2}\right\rangle^{2}$. If the $u$ are Gaussian, this number is three. For the purposes of a thought experiment, one may assume any $K \geq 1$. 
One must also consider the correlation, if any, amongst the $\omega_{m}$. It is widely argued that realistic reverberant structures have the natural frequency correlations of the GOE (Weaver, 1989a; b; Davy, 1990; 2009). Monsef et al. take in their theory the older and simpler assumption that the natural frequencies are uncorrelated. They implicitly also take the spectral density of eigenfrequencies $n$ to be constant, and all modes to have identical absorption rates $\gamma$. While those choices are arguably unphysical, they remain acceptable for the purposes of a thought experiment or for Monte Carlo simulations.

These assumptions about the $u$ and the natural frequencies may be - and often ought to be relaxed for application to real reverberant structures, but the present purposes do not require it.

Having described the statistics of the constituents $u$ and $\omega_{m}$ and $\gamma_{m}$ in the expression for $p$, it should be straightforward to then calculate the mean square of $|p|$ and the mean fourth power of $|p|$. The quantity of particular interest is the relative variance

$$
\operatorname{Relvar}=\frac{<\left(|p(\mathbf{x}, \omega)|^{2}-<|p(\mathbf{x}, \omega)|^{2}>\right)^{2}>}{<|p(\mathbf{x}, \omega)|^{2}>^{2}}=\frac{<|p(\mathbf{x}, \omega)|^{4}>}{<|p(\mathbf{x}, \omega)|^{2}>^{2}}-1
$$

which by construction is positive semi-definite. Theory in the literature has long agreed that, under the condition that the natural frequencies are uncorrelated, this quantity is $1+K^{2} / M$ where $M$ is the modal overlap which equals $2 \pi n \gamma$. Monsef et al. calculate that Relvar is $1+\left(K^{2}-2\right) / M$. That this result is impossible is readily recognized; Monsef et al.'s expression can take negative values for admissible values of $K$ and $M$. For example, consider the case that all $u^{2}=1$. Then $K=1$ and Relvar=1-1/M is negative for small $M$.

That others obtain similar looking but approximate expressions like $1+\left(K^{2}-3\right) / M$ for relative variance in the presence of natural frequency correlations is not in violation of the requirement that Relvar be nonnegative, because such expressions are derived under an assumption of high overlap $M$ and are not intended to apply at small $M$. 


\section{A KEY QUANTITY FOR THE THEORY OF Relvar}

It behooves us to determine how Monsef et. al. made their error. The error is subtle and occurs in their Appendix where they evaluate the expectation

$$
E_{\left.|\psi|^{\mid}|| \psi\right|^{2}}=<\sum_{m \neq k} \frac{1}{\left|\omega-\omega_{m}-i \gamma\right|^{2}\left|\omega-\omega_{k}-i \gamma\right|^{2}}>
$$

If the eigenfrequencies are uncorrelated (as assumed here and purportedly by Monsef et al.) the two factors under the summation are uncorrelated.

$$
E_{\left.|\psi||| \psi\right|^{2}}=\sum_{m \neq k}<\frac{1}{\left|\omega-\omega_{m}-i \gamma\right|^{2}}><\frac{1}{\left|\omega-\omega_{k}-i \gamma\right|^{2}}>
$$

The expectation becomes (Davy, 1981)

$$
E_{|\psi|^{2}|\psi|^{2}}=J(J-1)<\frac{1}{\Omega} \int \frac{d \omega_{m}}{\left|\omega-\omega_{m}-i \gamma\right|^{2}}>^{2}=\left(1-\frac{1}{J}\right) \frac{n^{2} \pi}{\gamma}
$$

where $J$ is the number of modes and $\Omega$ is the frequency range (such that modal density $n=J / \Omega$ ). On recognizing that $1 / J$ is negligible one obtains the standard result and, after a bit more algebra, the result Relvar $=1+K^{2} / M$. It is noteworthy that to derive this one must take each of the $J$ eigenfrequencies to range uniformly and independently in the ensemble over the full spectrum $\Omega$.

Monsef et al proceed differently. In their average of equation (4) over the ensemble of possible values for $\omega_{m}$ and $\omega_{k}$, they take each eigenfrequency to vary randomly and independently over a range (of width $\Delta \omega$ which must be less than a quantity of order $\gamma$ in order to justify the approximation used later to obtain their equation (A4) from their equation (A3)) centered on mean values that are indicated here by overbars, $\overline{\omega_{m}}$ and $\overline{\omega_{k}}$ respectively. In that case their evaluation of the sum in equation (4) is dominated by those few $k$ and $m$ such that $\overline{\omega_{m}}$ and $\overline{\omega_{k}}$ are near the working frequency $\omega$. Thus their effective $J$ is not large, but rather of order 
$M=2 \pi n \gamma$. In lieu of Davy's $1-1 / J$, they obtain $1-1 / M$; the consequences for Relvar are then as they describe.

So which is the correct form for the distribution of an eigenfrequency? Davy distributes $\omega_{m}$ over a long range $\Omega>>1 / n$. Monsef et al. distribute it over a narrow range around some mean. If the modes are ordered conventionally, by increasing value, such that $\omega_{m}$ is the $m^{\text {th }}$ lowest eigenfrequency, then as Monsef et al. imply, the $m^{\text {th }}$ eigenfrequency will have some mean value ${ }_{m}^{-}$(of order $\left.m / n\right)$, and be distributed over some limited range around that mean. If one takes the eigenfrequencies to be independently distributed and then ordered, the standard deviation of ${ }_{m}$ will be of order $\Delta \omega=m^{1 / 2} / n$, and not necessarily smaller than $\gamma$ as Monsef et al. required. Moreover, if the eigenfrequencies are chosen independently and then ordered there will be further correlations: the $m^{\text {th }}$ mode has precisely $m-1$ modes with lower frequency. (In Monsef's model the correlations are a bit weaker, than that.) In consequence, Monsef et al.'s decomposition of the sum in equation (3) to derive equation (4) becomes invalid. It may be possible to employ conventional ordering for calculations of Relvar, but the resulting correlations between $\omega_{k}$ and $\omega_{m}$ would make the calculation difficult. It is much simpler to eschew conventional ordering, as it serves no useful purpose. Davy's formulation in equation (5) is the correct one, with $1 / J$ negligible. It is also much simpler.

Monsef's assumed distribution of $\omega_{m}$ corresponds to high spectral rigidity. Their spectrum is highly uniform; a range $\delta \omega$ of their spectrum has an expected number of eigenfrequencies $n \delta \omega$, with a fixed variance that their model takes to be a constant proportional to the square of their quantity $\Delta \omega$. Poisson distributions have number variances (Brody et al., 1981) that grow linearly with range $\delta \omega$. The GOE has a number variance that grows logarithmically with range 
$\ln (\delta \omega)$ (Brody et al., 1981). Inasmuch as Monsef et al.'s variance is even less than the GOE's at long range, one may characterize their spectrum as having high spectral rigidity. As is well known, GOE rigidity leads to a prediction, at large enough $M$, that Relvar is $1+\left(K^{2}-3\right) / M$, so it is not surprising that Monsef et al.'s rigidity might lead to something similar.

\section{NUMERICAL RESULTS}

Monsef et al. admit that many readers might be skeptical that their expression $1+\left(K^{2}-2\right) / M$ could be correct, because of the long established $1+K^{2} / M$. In order to remove doubts, they present the results of a Monte Carlo (MC) simulation of equation (1) with Gaussian random real $u$ (so that $K=3$ ) and purportedly uncorrelated natural frequencies. Their MC results appear to be in agreement with $1+\left(K^{2}-2\right) / M$. It is not obvious why it agrees, but it is probable that their MC ensemble of natural frequencies contained a good deal of spectral rigidity. They appear to consider a base distribution of natural frequencies that is a "picket fence" in which all frequencies are separated by the same amount $1 / n$. They then perturb it randomly by smearing the distribution of each natural frequency over a finite range (taken to be $2 \pi / n$ ). This is consistent with our description above of their theory. If so, one can see why their MC numerical results corresponded so well to $1+\left(K^{2}-2\right) / M$ and not $1+K^{2} / M$. Their MC natural frequencies were in fact well correlated, with significant rigidity. Rigidity for the GOE case implies Relvar is $1+\left(K^{2}-3\right) / M$ at large $M$ (Weaver, 1989a), i.e. $1+6 / M$ for Gaussian mode shapes with $K=3$. That this is not very different from their theory $1+7 / M$ suggests that their spectral rigidity is responsible for the numerical agreement. In short, their MC spectrum was not Poissonian.

\section{OTHER ERRORS}

There are two other points unrelated to the discrepancy between $1+K^{2} / M$ and $1+\left(K^{2}-2\right) / M$, that ought to be mentioned. 
Monsef et al. make a claim about the approximation introduced by Weaver (1989a) in which the effect of rigidity was - at least for large enough $M$ - simply and analytically accounted for by taking the $Y_{2}$ function equal to a Dirac delta (Monsef $e t$ al.'s equation (8)). They claim that doing so is equivalent to the case of uncorrelated natural frequencies with a caveat that no pair of frequencies can be identical. That is an incorrect characterization; it would be correct only if $Y_{2}$ was replaced by a Kronecker delta.

Monsef et al. claim that their integral (25) converges, even if one inserts the usual threedimensional modal density (their equation (24)). This is incorrect. Their $|\psi|^{2}$ diminishes asymptotically inversely with the square of the frequency $f$ (not the fourth power like they claim); the modal density increases with the square of the frequency. Their integrand is therefore asymptotically independent of frequency and their integral does not converge. The error here is

merely one of exposition; $|\psi|^{2}$ would diminish like $f^{-4}$ if they had not already imposed the otherwise convenient approximation that the working frequency is of the same order as the relevant eigenfrequencies. Their integral (25) would converge using an unapproximated $\psi$, but not for the approximate $\psi$ as written in their equation (13). On replacing $n\left(\omega_{n}\right)$ with a constant $n\left(\omega_{w}\right)$ (as do most others) their integral again converges even with the approximate $\psi$. The net error is negligible in practice.

\section{CONCLUSIONS}

Our familiar and long-established and accepted expressions for relative variance when there is no decay curvature and no spectral rigidity need not be re-examined. The canonical work by Lyon and Davy does not need rehabilitation. This does not however mean that the question of power transmission variances is settled. The effects of decay curvature, of complex modes and 
finite GOE correlation range, with the consequent uncertainty as to the best choice for $K$ remain unclear.

\section{REFERENCES}

Brody, T. A., Flores, J., French, J. B., Mello, P. A., Pandey, A., and Wong, S. S. M. (1981). "Random Matrix Physics: Spectrum and strength fluctuations," Rev. Mod. Phys. 53, 385-478.

Burkhardt, J., and Weaver, R. L. (1996). "The effect of decay rate variability on statistical response predictions in acoustic systems," J. Sound Vib. 196, 147-164.

Davy, J. L. (1981). "The relative variance of the transmission function of a reverberation room," J. Sound Vib. 77, 455-479.

Davy, J. L. (1986). "The ensemble variance of random noise in a reverberation room," J. Sound Vib. 107, 361-373.

Davy, J. L. (1987). "Improvements to formulas for the ensemble relative variance of random noise in a reverberation room," J. Sound Vib. 115, 145-161.

Davy, J. L. (1990). "The distribution of modal frequencies in a reverberation room," in Science for Silence-Proceedings of Inter-Noise 90 Conference, edited by H. G. Jonasson (Gothenburg, Sweden), pp. 159-164.

Davy, J. L. (2009). "The variance of the discrete frequency transmission function of a reverberant room," J. Acoust. Soc. Am. 126, 1199-1206.

Jacobsen, F. (2012). "A note on the modal kurtosis and the concentration factorin reverberation rooms," J. Acoust. Soc. Am. 131, 2565-2569.

Langley, R. S., and Brown, A. W. M. (2004a). "The ensemble statistics of the band-averaged energy of a random system," J. Sound Vib. 275, 847-857. 
Langley, R. S., and Brown, A. W. M. (2004b). "The ensemble statistics of the energy of a random system subjected to harmonic excitation," J. Sound Vib. 275, 823-846.

Langley, R. S., and Cotoni, V. (2005). "The ensemble statistics of the vibrational energy density of a random system subjected to single point harmonic excitation," J. Acoust. Soc. Am. 118, 3064-3076.

Legrand, O., Mortessagne, F., and Sornette, D. (1995). "Spectral rigidity in the large modal overlap regime - beyond the Ericson-Schroeder hypothesis," Journal De Physique I 5, 10031010.

Lobkis, O. I., Weaver, R. L., and Rozhkov, I. (2000). "Power variances and decay curvature in a reverberant system," J. Sound Vib. 237, 281-302.

Lyon, R. H. (1969). "Statistical analysis of power injection and response in structures and rooms," J. Acoust. Soc. Am. 45, 545-565.

Monsef, F., Cozza, A., Rodrigues, D., Cellard, P., and Durocher, J.-N. (2014). "Relative variance of the mean-squared pressure in multimode media: Rehabilitating former approaches," J. Acoust. Soc. Am. 136, 2621-2629.

Rozhkov, I., Fyodorov, Y. V., and Weaver, R. L. (2004). "Variance of transmitted power in multichannel dissipative ergodic structures invariant under time reversal," Physical Review E 69, 1-10.

Weaver, R. L. (1989a). "On the ensemble variance of reverberation room transmission functions, the effect of spectral rigidity," J. Sound Vib. 130, 487-491.

Weaver, R. L. (1989b). "Spectral statistics in elastodynamics," J. Acoust. Soc. Am. 85, 10051013. 